\title{
Natural products as source of molecules with biological activity
}

The last two centuries will be remembered by the growth of knowledge about the natural world. The inquiry on the atom structure and the progress in the comprehension of electricity and magnetism opened doors towards the knowledge of our environment as humanity has never experienced before. Biology did not stay behind in this process and, because it is a descriptive discipline, it drew the most audacity hypotheses to decipher the past and present of living organisms. Today we are seeing and living with the effects of this accumulation of knowledge.

Where the progress of biology, chemistry and physics came together, was in the explanation of the effects of certain species, especially vegetables, on other living organisms, especially on mammals, and particularly on humans. Thus, the knowledge of medicinal preparations, poisons for hunting, magical preparations, dyes and other resources of ancient civilizations, was going through the sieve of scientific inspection seeking for validation or rejection, and in the first case to recognize molecules responsible for the proven effects.

Traditional medicine provided many valuable clues that led to the discovery of biologically active molecules, which eventually became medicine, and cases abound in the history of medicinal chemistry as the glycosides from Digitalis spp. to treat heart failure, or America's quinine or China's artemisinin to treat malaria. Experiences like these led us to consider indigenous pharmacopoeia as a valuable source to guide the search for novel molecules with biological activities. The titanic experience of the National Cancer Institute $(\mathrm{NCl})$, which after examining more than one hundred thousand extracts, yielded only two molecules to reach clinical applications which consolidated the bioprospecting approach based on traditional use.

However, relationships among species are extremely complex in nature, which exceed the assessments of cultures for their observation and understanding, and also because they were more focused on unknown pathologies by ancestral cultures. This situation led to the expansion to the search for active molecules in species with no history of traditional use, since they produce metabolites necessary for successful adaptation to their habitats. Molecules produced as part of metabolic processes have a high probability of interacting with biological structures, such as enzymes, receptors, or nucleic acids, and therefore manifest biological activity.

Despite the enormous development of other disciplines to generate alternative therapies such as monoclonal antibodies and the expression of products in genetically modified organisms, nature continues to provide directly, active molecules as such - or indirectly, structural leaders that led to clinically useful molecules - more than $40 \%$ of novel drugs that reached the market in the last half century.

Humans, even in countries in the process of achieving greater development, have been losing contact with nature that our ancestors used to have. In Latin America, we experienced rapid and extensive urbanization, often accompanied by productive practices in which income monocultures replaced much of the natural environment. In this process, and without assessing the risk, we have consented voluntarily or not to practices that led to the risk of biodiversity reduction. Unfortunately, when this topic is discussed, little attention is put on the loss of species that do not particularly inspire affection, such as birds or mammals, or that appeal for their color, like plants with showy flowers. Organisms such as plants and micro or macroscopic fungi developed a huge and varied synthetic capacity that allows them to adapt to environmental conditions. Species extinction, whatever its nature but with an emphasis on vegetables, 
carries the potential disappearance of a non-sizable number of novel molecules, and most likely bioactive, which is particularly foolish, as only a very small fraction of known plants, without the enormous diversity of microorganisms, insects and marine organisms, have been examined in chemical and biological terms in search of promising molecules. This consideration should support policies addressed to conserve biodiversity.

Prospection of active molecules based on purely chemical analysis followed by bioassays, the test of only species of traditional medicine or chemotaxonomic approach gave way to more current strategies seeking more effectiveness than in the past, and have given way to bioassay-guided isolation, generation of libraries of natural products, dereplication, metabolomic approaches and biotransformation in the effort to identify molecules that allow us to understand mechanisms of plant defense and apply this knowledge to elucidate molecular mechanisms and discover structures applicable in the therapeutic or phytosanitary protection, to name only two very motivating areas of scientific efforts.

The diverse flora of our country, in addition to a rich tradition of using nature's resources by all ethnic groups that still inhabit it, is a wealth that attracts research, and the threats to biodiversity justify that state, private, academia and business dedicate efforts to gain as much knowledge as possible as time gives us the opportunity to reveal this world of communication between and within species that expresses the chemistry of natural products.

Prof. Dr. Esteban A. Ferro Bertolotto Decano de la Facultad de Ciencias Quimicas Universidad Nacional de Asunción 